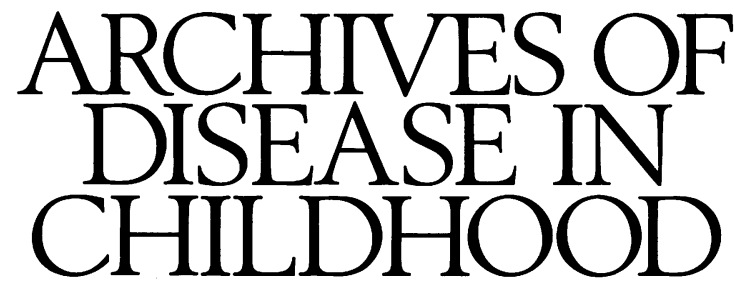

The fournal of the British Paediatric Association

\title{
Respiratory problems and cystic adenomatoid malformation of lung
}

Congenital cystic disease of the lung accounts for about $25 \%$ of lung malformations, and is an uncommon but important cause of neonatal respiratory distress. Congenital cystic disease includes: (1) lobar emphysema, which tends to affect a single upper lobe, is due to the obstruction of a lobar bronchus, and is consequent upon an abnormality in the bronchial cartilage allowing bronchial collapse and distal overinflation. (2) Bronchogenic cysts, which are unilocular and lined with ciliated respiratory epithelium, and are usually adjacent to major bronchi but do not communicate with them. They probably represent a malformation developing at the time of closure of the primitive foregut. (3) Pulmonary lymphangiectasia, which is rare, usually bilateral, and is due to congenital cystic dilatations of the pulmonary lymphatics. (4) Congenital cystic adenomatoid malformation of the lung, which tends to affect the lower lobes, and is thought to be due to an excessive overgrowth of terminal bronchiolar structures (that is, a hamartoma), and is lined with ciliated columnar epithelium but without any systematic bronchial architecture present. There is a wide range, varying from lobes containing cysts with minimal or no adenomatosis (more common, and may result from an embryonic defect occurring before 49 days' gestation) to adenomatosis with infrequent cysts (rare, and probably due to a defect occurring before 26 days' gestation).

\section{Presentation}

Patients with cystic adenomatoid malformation of the lung present with varying degrees of respiratory difficulty. Eighty percent of them present in the neonatal period with respiratory distress, often within hours of birth, and $14 \%$ are stillborn, ${ }^{1}$ Only $10 \%$ present after the first year of life, and they do so because of recurrent respiratory infections.

Neonates with the tachypnoea and dyspnoea of respiratory distress require urgent investigation to identify the cause. Cardiac abnormalities should be considered, and a chest radiograph must be taken to differentiate between hyaline membrane disease, infection, or a congenital malformation (for example, congenital diaphragmatic hernia or congenital lung malformation).

Cystic spaces seen in the chest on radiography initially raise suspicion of a congenital diaphragmatic hernia, with loops of bowel containing gas in the chest, as this is the most common diagnosis. It is therefore essential that a chest radiograph is taken with a nasogastric tube in situ, with enough abdomen visible on the radiograph to be able to see the full length of the tube, so that oesophageal displacement suggesting mediastinal shift, the position of the stomach, and the site of intestinal gas can be identified. However, cystic adenomatoid malformation can be differentiated on radiography by the presence of a normally situated stomach and intestine within the abdomen.

Cystic spaces in the chest can occur in association with infection, for example post staphylococcal pneumatocoeles. This diagnosis can be identified with the help of a reliable history and serial radiography.

The correct identification of cystic spaces in the chest

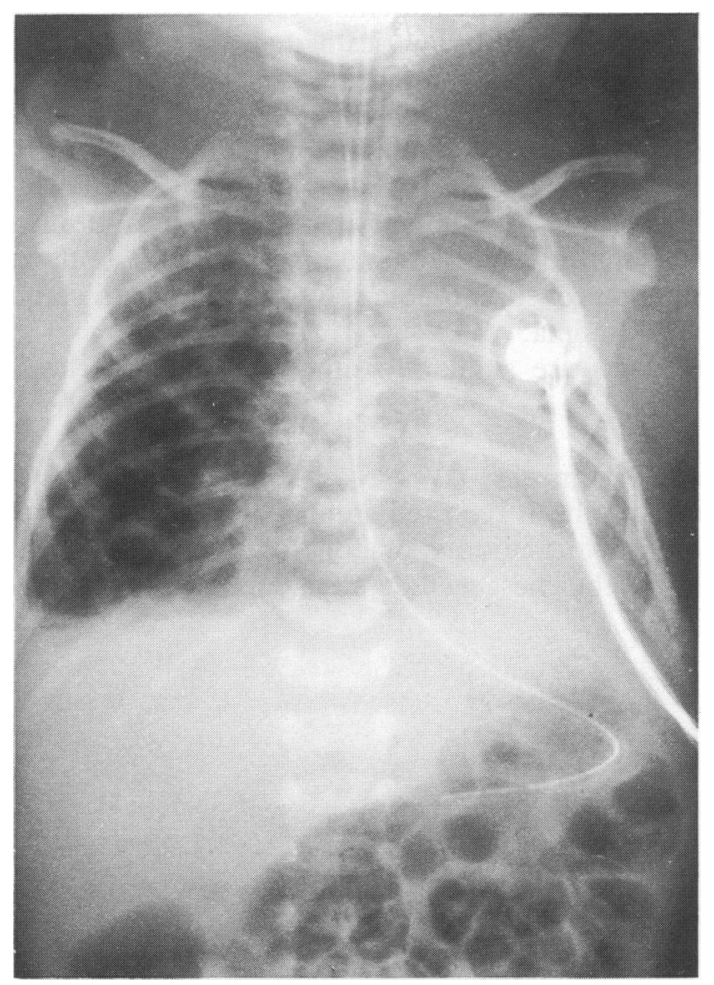

Radiograph of chest and upper abdomen demonstrating multiple cystic spaces in the right lung with mediastinal shift to the left, with a nasogastric tube entering a normally situated stomach with normal distribution of bowel gas and liver shadow. 
being due to cystic adenomatoid malformation of the lung requires awareness of the diagnosis and exclusion of other possible causes.

\section{Treatment}

Immediate management of a patient with cystic adenomatoid malformation is by appropriate treatment of the respiratory distress. The cystic spaces within the abnormal lobe will continue to increase in size as they aerate further. Morbidity is due to pressure of the expanded affected lobe onto the ipsilateral normal lobes and associated mediastinal shift with compression of the contralateral normal lung. Delay in diagnosis and hence appropriate treatment increases pressure symptoms, and clinical signs worsen. Respiratory distress may become severe and the baby may require supportive ventilation. The cysts are very prone to secondary infection, and this requires recognition and appropriate treatment.

Definitive treatment of cystic adenomatoid malformation of the lung is by thoracotomy and excision of the affected lobe or lobes. It is therefore essential to differentiate between congenital diaphragmatic hernia and cystic adenomatoid malformation of lung preoperatively in order to avoid an unnecessary laparotomy, which has happened, even in our own series.

In the last 36 years (1954-89) at this hospital, 16 neonates have undergone operative treatment for congenital cystic adenomatoid malformation of the lung. Three of these presented in September 1986, but no predisposing factors for this clustering have been identified. One of these patients was transferred to us with the diagnosis of congenital diaphragmatic hernia, but a repeat chest and abdominal radiograph (figure) with a nasogastric tube in situ enabled us to make the correct diagnosis before operation.

All these 16 patients (nine boys and seven girls) were born at term and presented with respiratory distress, nine within hours of birth, two in the first week, and the remaining five within four weeks. The patients were all treated surgically. Two patients early in the series had an initial inappropriate laparotomy, followed by a thoracotomy and lobectomy, and the other 14 patients had thoracotomy and excision of the affected lobe-the lower lobes being the most common (left $n=8$, right $n=4$ ). The left upper lobe was affected in one patient, the right middle lobe in one patient, and one died who was found to have had total involvement of both lungs. One patient proved subsequently to have cystic staphylococcal pneumonia.

These facts are similar to other published series. ${ }^{12}$

\section{Antenatal diagnosis}

Diagnosis has been reported antenatally, ${ }^{3-6}$ and cystic adenomatoid malformation is one of the differential diagnoses of a mass in the chest seen on an antenatal ultrasound scan.
Location of the stomach will particularly help in differentiating between cystic adenomatoid malformation and congenital diaphragmatic hernia.

As with all antenatal diagnoses of congenital malformations, it is important as it allows time for preparation and advice to the parents, and better planning of the place of delivery and subsequent management and treatment.

\section{Prognosis}

Neonates tolerate lobectomy extremely well, and follow up of our three recent patients with isotope studies shows that the remaining lung grows normally and expands to fill the thoracic cavity (unlike patients with repaired congenital diaphragmatic hernia where many of the lungs have a degree of hypoplasia). Clinically the lung functions well, although respiratory function tests may be expected to show a diminution of function.

\section{Conclusions}

Congenital cystic adenomatoid malformation of the lung can present with respiratory distress in the newborn. An awareness of the diagnosis is essential for all physicians involved in the care of neonates. All babies with cystic appearances on their chest radiography should have a radio-opaque nasogastric tube passed and a radiograph taken that includes the chest and abdomen in order to localise the oesophagus, stomach, and bowel gas to avoid mistaking the condition for a diaphragmatic hernia and hence the possibility of an inappropriate laparotomy.

The passage of time increases the problems caused by pressure from the aeration of the cystic lung with mediastinal shift making this condition a surgical emergency, and urgent referral should be made to a regional paediatric surgical centre.

J WALKER, R E CUDMORE

Department of Surgery

Alder Hey Children's Hospital

Eaton Road, Liverpool L12 2AP

1 Miller RK, Sieber WK, Yunis EJ. Congenital adenomatoid malformation of the lung: a report of 17 cases and review of the literature. Pathol Annu 1980; 15:387-402

2 Madewell JE, Stocker JT, Korsower JM. Cystic adenomatoid malformation of the lung: morphological analysis. American fournal of Roentgenology 1975; 124:436-48.

3 Cave APD, Adam AE. Cystic adenomatoid malformation of the lung found on antenatal ultrasound. $\mathrm{Br} \mathcal{F}$ Radiol 1984;57:176-8.

4 Johnson JA, Rumack CM, Johnson ML, Shikes R, Appareti K, Rees G. Cystic adenomatoid malformation: antenatal demonstration. American fournal of Roentgenology 1984;142:483-4.

5 Stauffer UG, Savoldelli G, Mieth D. Antenatal ultrasound diagnosis in cystic adenomatoid malformation of the lung - case report. $\mathcal{F}$ Pediatr Surg 1984; 19:141-2.

6 Morcos SF, Lobb MO. The antenatal diagnosis by ultrasonography of type III congenital cystic adenomatoid malformation of the lung. $\mathrm{Br} F$ Obstet Gynaecol 1986;93:1002-5. 